\title{
molecules
}

ISSN 1420-3049

(C) 2007 by MDPI

www.mdpi.org/molecules

\author{
Full Paper
}

\section{Synthesis of Novel Nitro-substituted Triaryl Pyrazole Derivatives as Potential Estrogen Receptor Ligands}

\author{
Fotini Naoum ${ }^{1}$, Konstantinos M. Kasiotis ${ }^{1}$, Prokopios Magiatis ${ }^{2}$ and Serkos A. Haroutounian ${ }^{1, *}$ \\ ${ }^{1}$ Chemistry Laboratory, Agricultural University of Athens, Iera odos 75, Athens 11855, Greece \\ ${ }^{2}$ Faculty of Pharmacy, Laboratory of Pharmacognosy and Natural Products Chemistry, University of \\ Athens, Panepistimiopolis Zografou, Athens 15771, Greece
}

* Author to whom correspondence should be addressed. E-mail: sehar@aua.gr

Received: 11 June 2007; in revised form: 29 June 2007 / Accepted: 29 June 2007 / Published: 2 July 2007

\begin{abstract}
Novel tetrasubstituted pyrazole derivatives bearing a nitro substituent on their A-phenol ring were synthesized and their binding affinity towards the estrogen receptor (ER) subtypes ER $\alpha$ and ER $\beta$ was determined. Among compounds tested, the 2-nitrophenol derivative 5c was found to bind satisfactorily to both estrogen receptor subtypes ( $R B A \alpha=5.17$ and $R B A \beta=3.27$ ). In general, the introduction of a nitro group into the A ring of these compounds was found to benefit their ER $\beta$ binding abilities.
\end{abstract}

Keywords: Pyrazoles, SERMs, Estrogen Receptor Binding Affinity.

\section{Introduction}

The estrogen receptor (ER) displays a considerable capacity for binding with a wide range of steroidal and nonsteroidal ligands. [1] Particularly, the nonsteroidal synthetic estrogens exhibit a tolerance in respect their structural motifs which can encompass, besides the phenolic function, which is a prerequisite for good binding, a large number of core building blocks. The latter may vary in length and size, including a plethora of -fused and nonfused- carbocyclic and heterocyclic ring systems [2-4]. 
An appreciable number of innovative Selective Estrogen Receptor Modulator compounds (SERMs) has been found to display a broad spectrum of agonist and antagonist actions at different target tissues. In addition, the further characterization of a second form of the receptor (ER $\beta$ subtype) [5-7], has focused the drug development research towards the discovery of ligands capable to activate or inhibit selectively each of the ER subtypes. For the development of novel SERMs a variety of diverse ligands has been considered.

Figure 1. 1,3,5-Triaryl-4-alkyl substituted pyrazole derivatives and 4-nitro-17 $\beta$-estradiol.

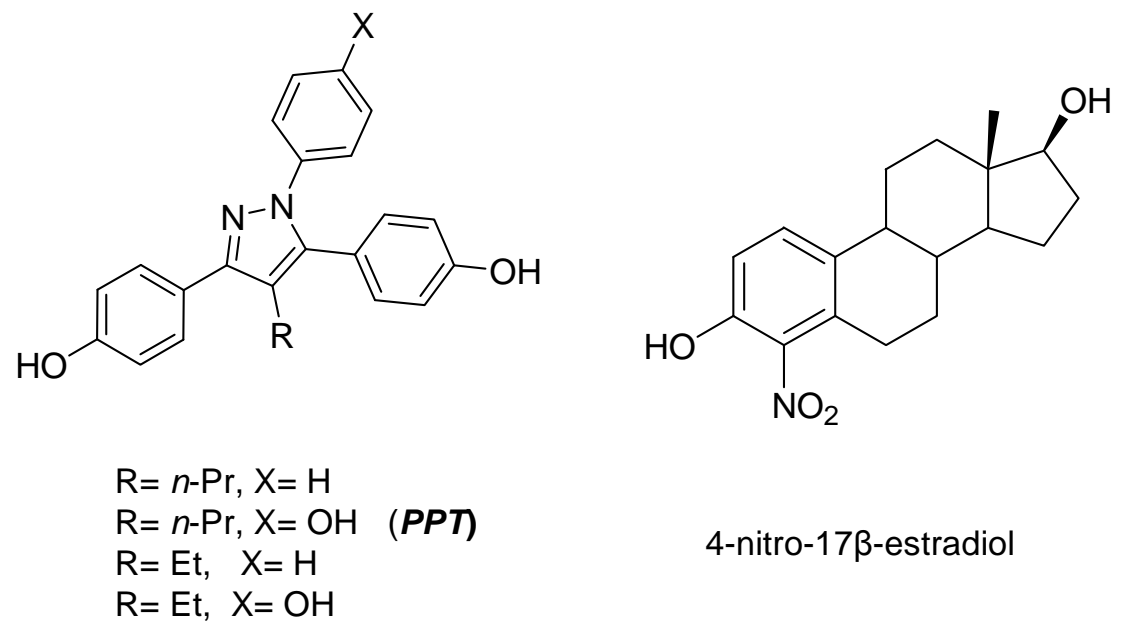

Among them, the tetrasubstituted pyrazole derivatives constitute an intriguing case, since 1,3,5triaryl-4-alkyl substituted pyrazoles, exemplified by the propylpyrazole triol PPT (Figure 1), have been found to possess particularly high ER $\alpha$-selective binding affinity and potency [8-10]. In this context, various research findings have indicated that the substitution pattern of these pyrazole derivatives greatly affects their binding affinity to the Estrogen Receptors [11].

In connection to our interest in ER binding selectivity pattern studies, we envisioned the synthesis of novel tetrasubstituted pyrazole derivatives bearing an $o$-nitro substituent on their A-phenol ring and possess the - critical for binding - alkyl group on $\mathrm{C}-4$ of the pyrazole ring. Recent reports have established that similar ring systems constitute valuable substrates for A-ring functionalised steroidal scaffolds [12] with potential application in asymmetric synthesis [13], supramolecular recognition [14], cancer chemotherapy [15], steroid receptor-based imaging [16] and material science [17]. In this regard, the 4-(and/or 2)-nitro-17 $\beta$-estradiol (Figure 1) have been exploited as compounds with anticancer activity and probes for ligand-receptor interaction studies [18-20]. Thus, we were interested in determining the impact of the presence of the electron-rich nitro group, as a peripheral substituent, on the ligand-receptor interaction of the propylpyrazole triol (PPT) analogue, that reduce the possible orientations of the A-phenol ring on the binding pocket of the ER. The design rational of these molecules is to combine in a single molecule the pharmacophore nitroestradiol ring with a tetra substituted pyrazole ring. 


\section{Results and Discussion}

\section{Chemistry}

The synthetic route to the target compounds includes the early stage preparation of the appropriate 1,3-diketones that were further used as substrates for the construction of the desired pyrazole substituted skeleta. In this regard, the starting alkylphenones 1a-c were prepared in good yields via the Friedel-Crafts acylation of anisole (Scheme 1).

Scheme 1. Synthesis of 1,3,5-triphenols 5a-d.
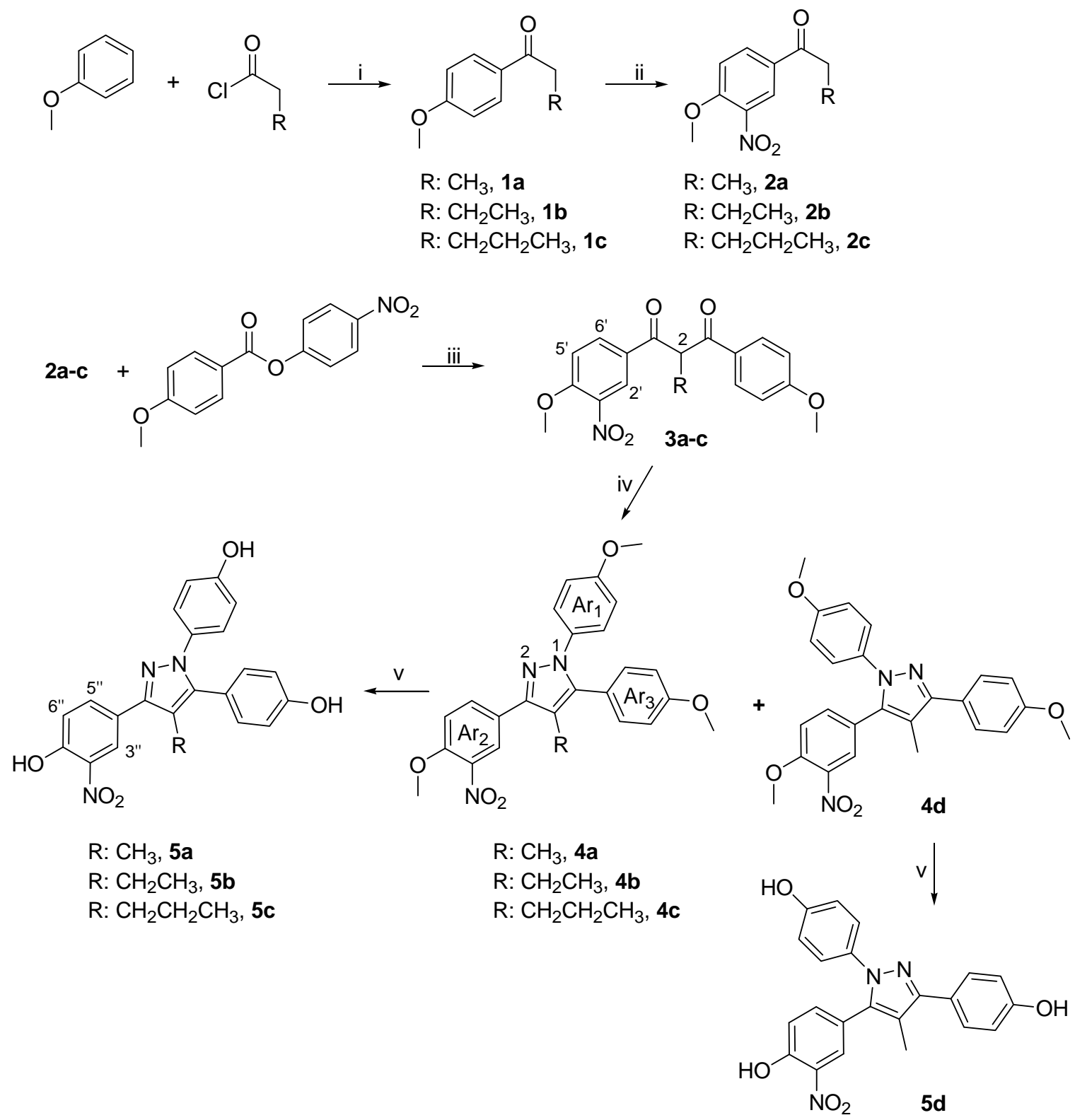

Reagents and conditions: (i) $\mathrm{AlCl}_{3}, \mathrm{CH}_{2} \mathrm{Cl}_{2}$; (ii) $\mathrm{KNO}_{3}, \mathrm{H}_{2} \mathrm{SO}_{4}$; (iii) LHMDS, THF; (iv) $\mathrm{CH}_{3} \mathrm{OArNHNH}{ }_{2} \cdot \mathrm{HCl}$, DMF / THF (3:1), $120^{\circ} \mathrm{C}$; (v) $\mathrm{BBr}_{3}, \mathrm{CH}_{2} \mathrm{Cl}_{2},-78^{\circ} \mathrm{C}$. 
Nitration of compounds 1a-c in sulfuric acid produced the nitrophenyl derivatives 2a-c, which were further alkylated to give $\beta$-diketones 3a-c. Double condensation of the latter with 4-methoxyphenylhydrazine hydrochloride provided the triarylpyrazole (4a-c) core ring system in satisfactory yields [21]. In the case of 2-methyl-propane-1,3-dione (3a) however, we also obtained the corresponding minor regioisomer $\mathbf{4 d}(\mathbf{4 a} / \mathbf{4 d}$ ratio $=4: 1)$, presumably because the attack from the opposite site is also possible in this case. This observation is partly in accordance with previously reported relative literature data [10]. The regioisomers obtained were separated by semi-preparative HPLC and their subsequent $\mathrm{BBr}_{3}$ promoted desmethylation provided the target phenolic pyrazoles 5a-d.

Scheme 2. Synthesis of 3,5-Diphenols $\mathbf{8 a}$ and $\mathbf{8 b}$.<smiles>[CH2+]CC(=O)c1ccc(OC)c([N+](=O)[O-])c1</smiles><smiles></smiles>

$2 a$<smiles>C#CC</smiles><smiles>COc1ccc(-n2nc(-c3ccc(OC)c([N+](=O)[O-])c3)c(C)c2-c2ccccc2)cc1</smiles><smiles>COc1ccc(-n2nc(-c3ccccc3)c(C)c2-c2ccc(OC)c([N+](=O)[O-])c2)cc1</smiles><smiles>Cc1c(-c2ccc(O)c([N+](=O)[O-])c2)nn(-c2ccc(O)cc2)c1-c1ccccc1</smiles><smiles>C#CC1CCCCC1</smiles><smiles>Cc1c(-c2ccccc2)nn(-c2ccc(O)cc2)c1-c1ccc(O)c([N+](=O)[O-])c1</smiles>

Reagents and conditions: (i) LHMDS, THF; (ii) $\mathrm{CH}_{3} \mathrm{OArNHNH}_{2} \cdot \mathrm{HCl}, \mathrm{DMF} / \mathrm{THF}(3: 1), 120^{\circ} \mathrm{C}$; (iii) $\mathrm{BBr}_{3}, \mathrm{CH}_{2} \mathrm{Cl}_{2},-78^{\circ} \mathrm{C}$.

The structure of the novel triaryl pyrazoles was determined by 2D-NOE spectroscopy experiments. More specifically, for compound $\mathbf{4 d}$, the observed enhancement of the deshielded aromatic protons of the phenylhydrazine moiety $\left(\mathrm{Ar}_{1}, 7.08 \mathrm{ppm}\right.$, doublet) with the $\mathrm{H}-6$ ' (resonates at $8.03 \mathrm{ppm}$ ) is 
indicative of the assigned regiochemistry. These results were further confirmed by gradient inverse detected long range ${ }^{1} \mathrm{H}-{ }^{15} \mathrm{~N}$ correlation experiments. Thus, for compound $4 \mathbf{d}$ the four bond correlation observed between the aromatic proton $\mathrm{H}_{-6}{ }^{\prime}(8.03 \mathrm{ppm})$ and the nitrogen atom $(\mathrm{N}-1)$ that resonates at $197.4 \mathrm{ppm}$ is an additional confirmation of the assigned structure. Furthermore, for compounds 4a-c the absence of NOE enhancement among the phenylhydrazine aromatic protons and the $\mathrm{H}-6$ ' of the nitro-substituted ring is indicative of the assigned regiochemistry. These results were also confirmed by gradient inverse detected long range ${ }^{1} \mathrm{H}-{ }^{15} \mathrm{~N}$ correlation experiments. In this regard the four bond correlation observed between the aromatic proton $\mathrm{H}-6$ ' (8.03 ppm) and the deshielded nitrogen atom $(\mathrm{N}-2)$ that resonates at $301.6 \mathrm{ppm}$ confirms the regiochemistry of compound $\mathbf{4 a}$.

The second pathway refers to the use of benzoyl chloride as acylating agent of 1-(4-methoxy-3nitro-phenyl)-propan-1-one (2a) to provide smoothly (Scheme 2) the diketone 6. The latter by condensation with 4-methoxyphenylhydrazine furnished an equimolar mixture of the regioisomers 7a,b. These derivatives were identified and analyzed after separation by semi-preparative HPLC as reported in the experimental part.

The structure of these isomers was also determined, through 2D-NOE spectroscopy experiments. More specifically, for compound $\mathbf{7 b}$, the observed strong cross peak among the deshielded phenylhydrazine group protons (multiplet at 7.37-7.14 ppm) with the $\mathrm{H}-6$ ' (resonates at $8.09 \mathrm{ppm}$ ) of the nitro-substituted ring is indicative of the assigned regiochemistry. These results were further verified by gradient inverse detected long range ${ }^{1} \mathrm{H}-{ }^{15} \mathrm{~N}$ correlation experiments. In this regard, a four bond correlation for compound $\mathbf{7 b}$ of the same aromatic proton $\mathrm{H}-6$ ' (8.09 ppm) and the shielded nitrogen atom ( $\mathrm{N}-1)$ that resonates at $199.8 \mathrm{ppm}$, was observed. According to the assigned structure the other nitrogen atom $(\mathrm{N}-2)$ resonates at $295.7 \mathrm{ppm}$ and correlates with the $\mathrm{N}$-attached aromatic protons and the $\mathrm{Ar}_{3} \mathrm{H}$. Finally the desired diphenols $\mathbf{8 a}$,b were obtained after ether cleavage with $\mathrm{BBr}_{3}$ in $\mathrm{CH}_{2} \mathrm{Cl}_{2}$ at $-78{ }^{\circ} \mathrm{C}$.

\section{ER Binding Affinity of Tetrasubstituted Pyrazoles to isolated human ER $\alpha$ and ER $\beta$}

The RBA values of the novel nitro triaryl pyrazole derivatives have been summarized in Table 1, reflecting the consequence of the steric interaction between the $\mathrm{C}(4)$-alkyl substituent and the nitro substituent of the A-ring. In this regard, compound 5c (PPT nitro analogue) was the most efficient, since was assayed to bind reasonably well to both ER $\alpha$ and ER $\beta$. As compared to previously reported results concerning PPT [8], its RBA $\beta$ binding ability has been increased by 27 fold, while a 9 fold decrease to ER $\alpha$ binding was also observed. Similar results were also obtained for the other triphenol derivatives tested (5b and 5d, 7 and 16 fold respective increse), indicating that the introduction of a nitro group into the A ring of these compounds force the neighboring alkyl group to an orientation that favours the ER $\beta$ binding. 
Table 1. Estrogen receptors (ER $\alpha$ and ER $\beta$ ) binding affinities of Novel Triaryl Pyrazoles (5a-d, 8a,b).<smiles>[X]c1ccc(-c2c([R])c(-c3ccc(O)c([N+](=O)[O-])c3)nn2-c2ccc(O)cc2)cc1</smiles>

5a-c, 8 a<smiles>[X]c1ccc(-c2nn(-c3ccc(O)cc3)c(-c3ccc(O)c([N+](=O)[O-])c3)c2[R])cc1</smiles>

$5 d, 8 b$

\begin{tabular}{|c|c|c|c|c|}
\hline Compound & $\mathbf{X}$ & $\mathbf{R}$ & $\operatorname{RBA} \mathbf{E R}^{\mathrm{a}}$ & RBA ER ${ }^{a}$ \\
\hline \multicolumn{5}{|c|}{ 1,3,5-Triphenols } \\
\hline $5 c$ & $\mathrm{OH}$ & $n-\mathrm{Pr}$ & $5.17 \pm 2.03$ & $3.27 \pm 0.04$ \\
\hline $5 b$ & $\mathrm{OH}$ & Et & $0.34 \pm 0.02$ & $0.83 \pm 0.18$ \\
\hline 5d & $\mathrm{OH}$ & $\mathrm{Me}$ & $0.49 \pm 0.33$ & $1.91 \pm 0.34$ \\
\hline $5 a$ & $\mathrm{OH}$ & $\mathrm{Me}$ & nd & nd \\
\hline \multicolumn{5}{|c|}{ 3,5-Diphenols } \\
\hline $8 a$ & $\mathrm{H}$ & $\mathrm{Me}$ & $0.74 \pm 0.30$ & $0.36 \pm 0.10$ \\
\hline $8 \mathbf{b}$ & $\mathrm{H}$ & $\mathrm{Me}$ & $0.53 \pm 0.16$ & $0.38 \pm 0.21$ \\
\hline PPT & $\mathrm{OH}$ & $n-\operatorname{Pr}$ & $49 \pm 12$ & $0.12 \pm 0.04$ \\
\hline
\end{tabular}

${ }^{\mathrm{a}}$ The RBA values (mean \pm SEM of at least three independent experiments) for ER $\alpha$ $(\mathrm{RBA} \alpha)$ and $\mathrm{ER} \beta(\mathrm{RBA} \beta)$ were calculated by [( $\mathrm{IC}_{50}$ estradiol / $\mathrm{IC}_{50}$ derivative) X 100], where $\mathrm{IC}_{50}$ values are estradiol and derivative concentrations capable of inhibiting binding of the fluorescent estrogen ES2 ( $1 \mathrm{nM})$ to ER $\alpha$ and ER $\beta$ by $50 \%$. IC ${ }_{50}$ values of estradiol for $\mathrm{ER} \alpha$ and $\mathrm{ER} \beta$ were $3.42 \pm 0.99$ and $2.87 \pm 0.64$, respectively. The RBA $\alpha$ and RBA $\beta$ of estradiol were set equal to 100. nd: non determined

\section{Experimental}

\section{General}

All anhydrous reactions were carried out under argon atmospheres. Solvents were dried by distillation prior to use. Solvent mixtures employed in chromatography were reported as volume to volume ratios. Starting materials were purchased from Aldrich (analytical reagent grades) and used without further purification. Analytical thin-layer chromatography (TLC) was conducted on Merck glass plates coated with silica gel $60 \mathrm{~F}_{254}$ and spots were visualized with UV light or/and an alcohol solution of anisaldehyde. Flash column chromatography was performed using Merck silica gel 60 (230-400 mesh ASTM). 
Melting points were determined on a Büchi melting point apparatus and are uncorrected. ${ }^{1} \mathrm{H}$ and 2D NMR spectra were recorded at $400 \mathrm{MHZ}$ on a Bruker DRX-400 spectrometer in the indicated solvents. The coupling constants are recorded in Hertz $(\mathrm{Hz})$ and the chemical shifts are reported in parts per million ( $\delta$, ppm) downfield from tetramethylsilane (TMS), which was used as an internal standard (by asterisk are indicated the overlapped peaks). For the ${ }^{1} \mathrm{H}-{ }^{15} \mathrm{~N}$ GHMQC spectrum, data were acquired as 3072 × 400 data points with a total of 290 transients accumulated/ $t_{1}$ increment. Pulse widths were $8.55 \mu$ s for ${ }^{1} \mathrm{H}$ and $27.7 \mu$ s for the ${ }^{15} \mathrm{~N}$ at powers of 0 and $-3 \mathrm{~dB}$. The $\mathrm{F} 1$ spectral window employed was set from 100 to 400 ppm. Pulsed field gradients, gt1-gt3, had durations of $0.8 \mathrm{~ms}$. Gradient pairs were optimized as 70:30:50 for ${ }^{15} \mathrm{~N}$.

Infrared spectra were obtained on a Nicolet Magna 750, series II spectrometer. HPLC separations were performed using a Hewlett Packard 1100 series instrument with a variable wavelength UV detector and coupled to HP Chem.-Station utilizing the manufacturer's 5.01 software package. A Kromasil column with pore size $100 \AA$, internal diameter of $5 \mu \mathrm{m}$, and a C-18 bond phase was used.

\section{General Procedure for the Preparation of Alkylphenones}

To an ice-cold solution of $\mathrm{AlCl}_{3}(46.8 \mathrm{mmol})$ in 1,2-dichloroethane $(10 \mathrm{~mL})$ an acid chloride (39.0 mmol) was added dropwise over $10 \mathrm{~min}$. The resulting solution was allowed to reach the room temperature and dissolve the $\mathrm{AlCl}_{3}$. Then, the reaction mixture was cooled to $0{ }^{\circ} \mathrm{C}$ and a solution of anisole $(46.8 \mathrm{mmol})$ in 1,2-dichloroethane $(20 \mathrm{~mL})$ was added dropwise over a period of $30 \mathrm{~min}$. Upon completion, the reaction was allowed to reach the room temperature and stirred for 8-15 h. The mixture was quenched by pouring over ice $(100 \mathrm{~g})$ and extracted with EtOAc $(3 \times 25 \mathrm{~mL})$. The combined organic layers were successively washed with water, $\mathrm{NaHCO}_{3}$ (satd), brine, dried over anhydrous $\mathrm{MgSO}_{4}$ and concentrated under reduced pressure. The residue was purified by flash column chromatography using a 5\% EtOAc/hexanes system as eluent.

\section{1-(4-Methoxy-phenyl)-propan-1-one (1a)}

Prepared according to the general procedure as outlined above affording, after flash chromatographic purification (5\% EtOAc/hexanes), the title compound was obtained as a pale yellow oil (5.61 g, 73\%). ${ }^{1} \mathrm{H}-\mathrm{NMR}\left(\mathrm{CDCl}_{3}\right) \delta, 7.91$ (dd, $\left.J=2.0,7.0 \mathrm{~Hz}, 2 \mathrm{H}, \mathrm{ArH}\right), 6.89$ (dd, $J=2.0,7.0 \mathrm{~Hz}$, 2H, $\mathrm{ArH}$ ), 3.83 (s, 3H, $\mathrm{OC}_{3}$ ), 2.91 (q, $J=7.4 \mathrm{~Hz}, 2 \mathrm{H}, \underline{\mathrm{C}}_{2} \mathrm{C}=\mathrm{O}$ ), 1.18 (t, $J=7.4 \mathrm{~Hz}, 3 \mathrm{H}, \underline{\mathrm{C}}_{3}$ ).

\section{1-(4-Methoxyphenyl)-butan-one (1b)}

Anisole (46.8 mmol) was reacted with butyl chloride $(39.0 \mathrm{mmol})$ according to the general procedure. Flash column chromatography (5\% EtOAc/hexanes) provided the title compound as a pale yellow oil (5.41 g, 65\%). ${ }^{1} \mathrm{H}-\mathrm{NMR}\left(\mathrm{CDCl}_{3}\right) \delta, 8.37$ (dd, $\left.J=2.0,7.0 \mathrm{~Hz}, 2 \mathrm{H}, \mathrm{ArH}\right), 7.34$ (dd, $J=2.0$, $7.0 \mathrm{~Hz}, 2 \mathrm{H}, \mathrm{ArH}), 4.28$ (s, 3H, $\mathrm{OC}_{3}$ ), 3.31 (t, $J=7.3 \mathrm{~Hz}, 2 \mathrm{H}, \underline{\mathrm{C}}_{2} \mathrm{C}=\mathrm{O}$ ), 2.18 (m, 3H, $\left.\underline{\mathrm{H}}_{2}\right), 1.41$ (t, $J$ $\left.=7.3 \mathrm{~Hz}, 3 \mathrm{H}, \underline{\mathrm{CH}}_{3}\right)$. 


\section{1-(4-Methoxyphenyl)-pentan-1-one (1c)}

Prepared from anisole $(46.8 \mathrm{mmol})$ and valeryl chloride $(39.0 \mathrm{mmol})$ following the general procedure, which afforded the title compound as a yellow oil (6.56 g, $73 \%) .{ }^{1} \mathrm{H}-\mathrm{NMR}\left(\mathrm{CDCl}_{3}\right) \delta, 7.91$

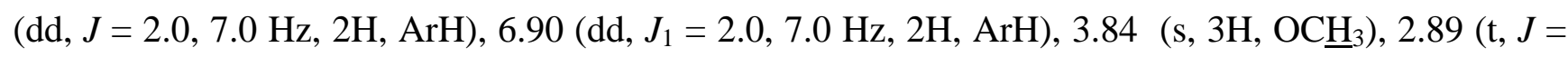
$6.2 \mathrm{~Hz}, 2 \mathrm{H}, \mathrm{C}_{2} \mathrm{C}=\mathrm{O}$ ), 1.68 (m, 2H, $\left.\mathrm{COCH}_{2} \underline{\mathrm{CH}}_{2}\right), 1.37$ (m, 2H, $\mathrm{COCH}_{2} \mathrm{CH}_{2} \mathrm{C}_{2}$ ), 0.92 (t, J = 7.4 Hz, $\left.3 \mathrm{H}, \mathrm{CH}_{3}\right)$.

\section{General Procedure for Nitration.}

To an ice-cold solution of 1a-c $(6.7 \mathrm{mmol})$ in $\mathrm{H}_{2} \mathrm{SO}_{4}(12.4 \mathrm{~mL})$ was added $\mathrm{KNO}_{3}(7.4 \mathrm{mmol})$ over a period of $30 \mathrm{~min}$. The reaction was run at room temperature for 8-15 h, quenched with $\mathrm{H}_{2} \mathrm{O}$ (15-25 $\mathrm{mL}$ ), extracted repeatedly with EtOAc ( $3 \times 25 \mathrm{~mL}$ ) and the combined organic layers were washed with a satd $\mathrm{NaHCO}_{3}$ solution (25 mL). The organic layer was separated, dried over anhydrous $\mathrm{MgSO}_{4}$ and concentrated under reduced pressure to afford the crude product as a white solid, which was purified by flash chromatography.

1-(4-Methoxy-3-nitro-phenyl)-propan-1-one (2a).

1a $(1.1 \mathrm{~g}, 6.7 \mathrm{mmol})$ was reacted with $\mathrm{KNO}_{3}(0.74 \mathrm{~g}, 7.4 \mathrm{mmol})$ according to the general nitration procedure, to give a dark yellow slurry. The latter was purified by flash chromatography (20\% EtOAc/hexanes) yielding a yellow solid (0.95 g, 68\%), which was recrystallized from $\mathrm{Et}_{2} \mathrm{O}$ to give pale yellow fine needles. M.p. 88-89 ${ }^{\circ} \mathrm{C}$; ${ }^{1} \mathrm{H}-\mathrm{NMR}\left(\mathrm{CDCl}_{3}\right) \delta, 8.41$ (d, $J=2.0 \mathrm{~Hz}, 1 \mathrm{H}, \mathrm{H}-2$ '), 8.15 (dd, $J=2.0,9.0 \mathrm{~Hz}, 1 \mathrm{H}, \mathrm{H}-6$ '), 7.13 (d, $J=9.0 \mathrm{~Hz}, 1 \mathrm{H}, \mathrm{H}-5$ '), 4.01 (s, 3H, OC${ }_{3}$ ), 2.97 (q, $J=7.4 \mathrm{~Hz}, 2 \mathrm{H}$, $\mathrm{C}_{2} \mathrm{CH}_{3}$ ), 1.20 (t, $J=7.4 \mathrm{~Hz}, 3 \mathrm{H}, \mathrm{CH}_{2} \underline{\mathrm{CH}}_{3}$ ); Elem. Anal.: C 57.41, H 5.30, N 6.70; C 57.59, H 5.42, N 6.52 .

\section{1-(4-Methoxy-3-nitro-phenyl)-butan-1-one (2b)}

Prepared according to general procedure for nitration from $\mathbf{1 b}(1.19 \mathrm{~g}, 6.7 \mathrm{mmol})$ and purified by flash chromatography (20\% EtOAc/hexanes) to afford a white solid (0.91 g, 61\%). M.p. 69-70 ${ }^{\circ} \mathrm{C}$; ${ }^{1} \mathrm{H}$ NMR $\left(\mathrm{CDCl}_{3}\right) \delta, 8.46$ (d, $J=2.2 \mathrm{~Hz}, 1 \mathrm{H}, \mathrm{H}-2^{\prime}$ ), 8.19 (dd, $J=2.2,8.8 \mathrm{~Hz}, \mathrm{H}-6$ '), 7.18 (d, $J=8.8 \mathrm{~Hz}$, H-5'), 4.06 (s, 3H, OC$\underline{H}_{3}$ ), 2.94 (t, $J=7.3 \mathrm{~Hz}, 2 \mathrm{H}, \mathrm{C}_{2} \mathrm{C}=\mathrm{O}$ ), 2.63 (m, 2H, $\underline{\mathrm{C}}_{2}$ ), 1.03 (t, $J=7.4 \mathrm{~Hz}$, 3H, $\underline{\mathrm{CH}}_{3}$ ); Elem. Anal.: C 59.19, H 5.87, N 6.27; C 59.39, H 5.72, N 6.41.

\section{1-(4-Methoxy-3-nitro-phenyl)-pentan-1-one (2c)}

Prepared according to general procedure for nitration as described above from 1c and purified by flash chromatography (20\% EtOAc/hexanes) to afford a white crystalline solid (1.0 g, 63\%). M.p. 81$82{ }^{\circ} \mathrm{C} ;{ }^{1} \mathrm{H}-\mathrm{NMR}\left(\mathrm{CDCl}_{3}\right) \delta, 8.40$ (d, $J=2.5 \mathrm{~Hz}, 1 \mathrm{H}, \mathrm{H}-2$ '), 8.14 (dd, $J=2.5,9.4 \mathrm{~Hz}, 1 \mathrm{H}, \mathrm{H}-6$ '), 7.12 (d, $J=9.4 \mathrm{~Hz}, 1 \mathrm{H}, \mathrm{H}-5$ '), 4.01 (s, 3H, OC$\underline{H}_{3}$ ), 2.91 (t, $J=7.4 \mathrm{~Hz}, 2 \mathrm{H}, \mathrm{CH}_{2} \mathrm{CH}_{2} \mathrm{CH}_{2} \mathrm{CH}_{3}$ ), 1.68 (m, 2H, $\mathrm{CH}_{2} \underline{\mathrm{C}}_{2} \mathrm{CH}_{2} \mathrm{CH}_{3}$ ), 1.37 (m, 2H, $\mathrm{CH}_{2} \mathrm{CH}_{2} \mathrm{CH}_{2} \mathrm{CH}_{3}$ ), 0.92 (t, $J=7.4 \mathrm{~Hz}, 3 \mathrm{H}, \mathrm{CH}_{2} \mathrm{CH}_{2} \mathrm{CH}_{2} \mathrm{C}_{3}$ ); Elem. Anal.: C 60.75, H 6.37, N 5.90; C 60.61, H 6.45, N 5.82. 
General Procedure for the Preparation of Diketones 3a-c

To an ice-cold solution of 2a-c (0.97 mmol) and 4-nitrophenyl-4-methoxy benzoate (0.97 mmol) [prepared from p-nitrophenol and 4-methoxybenzoic acid using diisopropylcarbodiimide and 4dimethylaminopyridine] in THF (35 mL), was added a $1.0 \mathrm{M}$ solution of lithium hexamethyldisilamide (3.03 $\mathrm{mmol}$ ) dropwise over a period of $5 \mathrm{~min}$. The reaction was allowed to reach the room temperature and stirred for additional $1.5 \mathrm{~h}$ and quenched with $\mathrm{H}_{2} \mathrm{O}(25 \mathrm{~mL})$. Then, the mixture was repeatedly extracted with $\mathrm{Et}_{2} \mathrm{O}$ and the combined organic layers were washed with $\mathrm{H}_{2} \mathrm{O}$, dried over anhydrous $\mathrm{Na}_{2} \mathrm{SO}_{4}$ and concentrated under reduced pressure to afford a crude solid. The unreacted ester was removed by the addition of a solution of $40 \%$ EtOAc/ hexanes and the filtration of the insoluble ester. The filtrate was concentrated in vacuo and purified by flash chromatography using various proportions of EtOAc/hexanes as eluent system.

1-(4-Methoxy-3-nitrophenyl)-3-(4-methoxyphenyl)-2-methyl-propane-1,3-dione (3a)

Prepared according to general procedure for preparation of diketones outlined above from 2a (0.6 g, $2.9 \mathrm{mmol}$ ) and purified by flash chromatography (20\% EtOAc/hexanes) to yield the title product as an orange oil (0.74 g, 74\%). ${ }^{1} \mathrm{H}-\mathrm{NMR}\left(\mathrm{CDCl}_{3}\right) \delta, 8.39$ (d, $J=2.2 \mathrm{~Hz}, 1 \mathrm{H}, \mathrm{H}-2$ ') 8.09 (dd, $J=2.2,8.4$ Hz, 1H, H-6'), 7.08 (d, $J=8.4$ Hz, 1H, H-5'), 7.92 (d , $J=8.9$ Hz, 2H, ArH), 6.93 (d, J= 8.9 Hz, 2H,

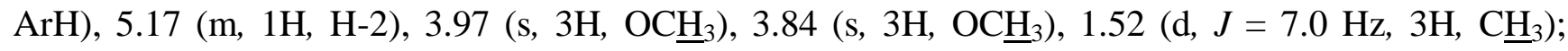
Elem. Anal.: C 62.97, H 4.99, N 4.08; C 62.71, H 4.81, N 4.19.

2-Ethyl-1-(4-methoxy-3-nitrophenyl)-3-(4-methoxyphenyl)-propane-1,3-dione (3b)

Ketone $2 \mathbf{b}$ (0.4 g, $1.8 \mathrm{mmol}$ ) reacted with 4-nitrophenyl 4-methoxybenzoate (1.8 $\mathrm{mmol})$ as above to afford after flash chromatographic purification (40\% EtOAc/hexanes) compound $3 \mathbf{b}$ as a dark yellow oil $(0.26 \mathrm{~g}, 68 \%)$, while $0.15 \mathrm{~g}$ of the ketone $\mathbf{2 b}$ remained unreacted. ${ }^{1} \mathrm{H}-\mathrm{NMR}\left(\mathrm{CDCl}_{3}\right) \delta, 8.46$ (d, $J=2.2$ Hz, 1H, H-2'), 8.19 (dd, $J=2.2,8.9$ Hz, 1H, H-6'), 7.99 (d, $J=8.9$ Hz, 1H, H-5'), 7.74 (d , $\left.J=8.9 \mathrm{~Hz}, 2 \mathrm{H}, \mathrm{Ar}_{2} \mathrm{H}\right), 6.97\left(\mathrm{~d}, J=8.9 \mathrm{~Hz}, 2 \mathrm{H}, \mathrm{Ar}_{2} \mathrm{H}\right), 4.97(\mathrm{t}, J=6.7 \mathrm{~Hz}, 1 \mathrm{H}, \mathrm{H}-2), 4.02(\mathrm{~s}, 3 \mathrm{H}$, $\mathrm{OC}_{3}$ ), 3.89 (s, 3H, $\mathrm{OC}_{3}$ ), 2.18 (m, $2 \mathrm{H}, \mathrm{C}_{2}$ ), 1.05 (t, $J=7.4 \mathrm{~Hz}, 3 \mathrm{H}, \underline{\mathrm{C}}_{3}$ ); Elem. Anal.: C 63.86, H 5.36, N 3.92; C 63.61, H 5.45, N 3.82.

1-(4-Methoxy-3-nitrophenyl)-3-(4-methoxyphenyl)-2-propylpropane-1,3-dione (3c)

Prepared according to general procedure for preparation of 1,3-diketones outlined above from 2c (0.71 g, $3.0 \mathrm{mmol}$ ) and purified by flash chromatography (50\% EtOAc/hexanes) to provide a red oil $(0.44 \mathrm{~g}, 67 \%)$ and $0.29 \mathrm{~g}$ of the unreacted ketone $2 \mathrm{c} .{ }^{1} \mathrm{H}$ NMR $\left(\mathrm{CDCl}_{3}\right) \delta, 8.47(\mathrm{~d}, J=2.2 \mathrm{~Hz}, 1 \mathrm{H}, \mathrm{H}-$ 2'), 8.20 (dd, $J=2.2,8.8 \mathrm{~Hz}, 1 \mathrm{H}, \mathrm{H}-6$ ') 7.97 (d, $J=8.8 \mathrm{~Hz}, 2 \mathrm{H}, \mathrm{Ar}_{2} \mathrm{H}$ ), 7.14 (d , $J=8.8 \mathrm{~Hz}, 1 \mathrm{H}, \mathrm{H}-$ 5'), 6.98 (d, $J=8.8 \mathrm{~Hz}, 2 \mathrm{H}, \mathrm{Ar}_{2} \mathrm{H}$ ), 5.05 (t, $\left.J=6.6 \mathrm{~Hz}, 1 \mathrm{H}, \mathrm{H}-2\right), 4.03$ (s, 3H, OC$\underline{H}_{3}$ ), 3.89 (s, 3H, $\left.\mathrm{OC}_{3}\right), 2.12$ (m, 2H, $\left.\underline{\mathrm{C}}_{2}\right), 1.46$ (m, 2H, $\left.\underline{\mathrm{C}}_{2}\right), 0.99$ (t, $\left.J=7.3 \mathrm{~Hz}, 3 \mathrm{H}, \underline{\mathrm{C}}_{3}\right)$. Elem. Anal.: C 64.68, H 5.70, N 3.77; C 64.80, H 5.65, N 3.82. 
1-(4-Methoxy-3-nitrophenyl)-2-methyl-3-phenylpropane-1,3-dione (6)

To a solution of 2a (1.21 g, $5.8 \mathrm{mmol})$ in THF (15 mL), a solution of LHMDS (5.8 mmol, 1M in THF) was added dropwise over 15 min. Upon complete addition of LHMDS, the reaction was stirred for additional $15 \mathrm{~min}$. Then, benzoyl chloride (5.8 mmol, in $3 \mathrm{~mL}$ THF) was added dropwise and the reaction was run under stirring for $12 \mathrm{~h}$. The reaction mixture was repeatedly extracted with $\mathrm{Et}_{2} \mathrm{O}$ and the combined organic layers were washed with $\mathrm{H}_{2} \mathrm{O}$, dried over $\mathrm{MgSO}_{4}$ and concentrated under reduced pressure to afford a crude solid, which was purified by flash chromatography eluting using $5 \%$ EtOAc/hexanes as solvent eluting system (1.34 g, 74\%). ${ }^{1} \mathrm{H}-\mathrm{NMR}\left(\mathrm{CDCl}_{3}\right) \delta, 8.41$ (d, $J=2.0 \mathrm{~Hz}, 1 \mathrm{H}$, H-2'), 8.25 (d, $J=9.4 \mathrm{~Hz}, 1 \mathrm{H}, \mathrm{H}-5^{\prime}$ ), 8.11 (dd, $J=2.0,9.4 \mathrm{~Hz}, 1 \mathrm{H}, \mathrm{H}-6$ '), 7.93 (t , $J=9.0 \mathrm{~Hz}, 2 \mathrm{H}$, ArH), 7.46 (m, $J=7.8 \mathrm{~Hz}, 1 \mathrm{H}, \mathrm{ArH}), 7.13$ (d, $J=9.0 \mathrm{~Hz}, 2 \mathrm{H}, \mathrm{ArH}), 5.19$ (q, $J=7.0 \mathrm{~Hz}, 1 \mathrm{H}, \mathrm{CHCH}_{3}$ ), 3.99 (s, 3H, $\mathrm{OC}_{3}$ ), 1.24 (d, $J=7.0$ Hz, 3H, $\underline{\mathrm{C}}_{3}$ ); Elem. Anal.: C 68.17, H 4.83, N 4.47; C 67.35, H 4.71, N 4.35 .

\section{General Procedure for Pyrazole Synthesis}

To a solution of diketone $(1.0 \mathrm{mmol})$ in DMF $(30 \mathrm{~mL})$ and THF (10 mL), 4-methoxyphenylhydrazine hydrochloride (4-5 equiv) was added. The mixture was brought to reflux and the reaction progress was monitored by TLC analysis $(10-20 \mathrm{~h})$. Then the reaction mixture was allowed to cool to room temperature and diluted with $\mathrm{H}_{2} \mathrm{O}(30 \mathrm{~mL})$. The product was repeatedly extracted with EtOAc ( $3 \times 25 \mathrm{~mL})$ and the combined organic layers and sequentially washed with a satd LiCl solution (25 mL), satd $\mathrm{NaHSO}_{3}(25 \mathrm{~mL})$, and brine $(25 \mathrm{~mL})$. The organic layer was dried over $\mathrm{Na}_{2} \mathrm{SO}_{4}$ and concentrated under reduced pressure to afford a crude oil, which was purified by flash chromatography using EtOAc/hexanes as eluting solvent system.

3-(4-Methoxy-3-nitrophenyl)-1,5-bis-(4-methoxyphenyl)-4-methyl-1H-pyrazole (4a) and 5-(4-methoxy3-nitrophenyl)-1,3-bis-(4-methoxyphenyl)-4-methyl-1H-pyrazole (4d)

Diketone 3a was reacted with 4-methoxyphenylhydrazine hydrochlorides as described above. Flash chromatographic purification (20\% EtOAc/hexanes) provided an inseparable mixture of the title compounds (68\%). The latter were separated by semipreparative HPLC separation using $\mathrm{H}_{2} \mathrm{O} / \mathrm{CH}_{3} \mathrm{CN}$ (15:85) as eluent in a flow rate of $1.6 \mathrm{ml} / \mathrm{min}$, furnishing the two regioisomers in a $4: 1$ ratio for $\mathbf{4 a}$ and 4d respectively ( $U V$ detector at $\left.254 \mathrm{~nm}, \mathrm{t}_{\mathrm{R} 4 \mathrm{a}}=12.2 \mathrm{~min}, \mathrm{t}_{\mathrm{R} 4 \mathrm{~d}}=15.4 \mathrm{~min}\right) . \mathbf{4 a}:{ }^{1} \mathrm{H}-\mathrm{NMR}\left(\mathrm{CDCl}_{3}\right) \delta, 8.32$ (d, $J=2.2 \mathrm{~Hz}, 1 \mathrm{H}, \mathrm{H}-2$ '), 8.03 (dd, $J=2.2,8.7 \mathrm{~Hz}, 1 \mathrm{H}, \mathrm{H}-6$ '), 7.71 (d, $J=8.7 \mathrm{~Hz}, 1 \mathrm{H}, \mathrm{H}-5$ '), 7.26 (d, $J=8.5 \mathrm{~Hz}, 2 \mathrm{H}, \mathrm{Ar}_{3} \mathrm{H}$ ), 7.14 (d, $\left.J=8.9 \mathrm{~Hz}, 2 \mathrm{H}, \mathrm{Ar}_{1} \mathrm{H}\right), 6.92$ (d, $\left.J=8.5 \mathrm{~Hz}, 2 \mathrm{H}, \mathrm{Ar}_{3} \mathrm{H}\right), 6.82(\mathrm{~d}, J=8.9$

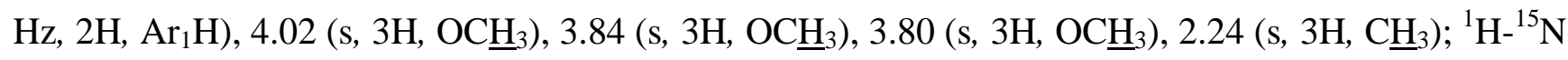
NMR $\left(\mathrm{CDCl}_{3}\right) \delta, \underline{\mathrm{N}-1}$ 205.6, $\underline{\mathrm{N}-2}$ 301.6; Elem. Anal.: C 67.41, H 5.20, N 9.43; C 67.25, H 5.41, N 9.32; 4d: ${ }^{1} \mathrm{H}-\mathrm{NMR}\left(\mathrm{CDCl}_{3}\right) \delta, 8.34$ (d, $J=2.1 \mathrm{~Hz}, 1 \mathrm{H}, \mathrm{H}-2$ '), 8.03 (dd, $J=2.1,8.8 \mathrm{~Hz}, 1 \mathrm{H}, \mathrm{H}-6$ '), 7.60 (d, $J=8.8 \mathrm{~Hz}, 1 \mathrm{H}, \mathrm{H}-5$ ') 7.22 (d, $J=8.5 \mathrm{~Hz}, 2 \mathrm{H}, \mathrm{Ar}_{3} \mathrm{H}$ ), 7.08 (d, $\left.J=8.7 \mathrm{~Hz}, 2 \mathrm{H}, \mathrm{Ar}_{1} \mathrm{H}\right), 6.92$ (d, $J=$ $8.5 \mathrm{~Hz}, 2 \mathrm{H}, \mathrm{Ar}_{3} \mathrm{H}$ ), 6.80 (d, $J=8.7 \mathrm{~Hz}, 2 \mathrm{H}, \mathrm{Ar}_{1} \mathrm{H}$ ), 4.04 (s, 3H, OC$\underline{\mathrm{H}}_{3}$ ), 3.85 (s, $3 \mathrm{H}, \mathrm{OC}_{3}$ ), 3.80 (s, $\left.3 \mathrm{H}, \mathrm{OC}_{3}\right), 2.16$ (s, 3H, $\left.\underline{\mathrm{CH}}_{3}\right) ;{ }^{1} \mathrm{H}^{15}{ }^{15} \mathrm{NMR}\left(\mathrm{CDCl}_{3}\right) \delta, \underline{\mathrm{N}-1}$ 197.4, $\underline{\mathrm{N}-2}$ 294.7; Elem. Anal.: C 67.41, H 5.20, N 9.43; C 67.54, H 5.32, N 9.30. 
Diketone 3b (0.23 g, $0.6 \mathrm{mmol})$ was reacted with 4-methoxyphenylhydrazine hydrochloride (0.53 g, $3.0 \mathrm{mmol}$ ) according to the general procedure. Upon purification by flash chromatography (40\% EtOAc/hexanes) the title compound was obtained as a tan solid $(0.21$ g, $75 \%)$. M.p. $121-122{ }^{\circ} \mathrm{C} ;{ }^{1} \mathrm{H}$ NMR $\left(\mathrm{CDCl}_{3}\right) \delta, 8.32$ (d, $J=2.2 \mathrm{~Hz}, 1 \mathrm{H}, \mathrm{H}-2$ '), 8.02 (dd, $J=2.2,8.7 \mathrm{~Hz}, 1 \mathrm{H}, \mathrm{H}-6$ '), 7.68 (d, $J=8.7$ $\mathrm{Hz}, 1 \mathrm{H}, \mathrm{H}-5$ '), 7.21 (dd, $J=2.0,8.8 \mathrm{~Hz}, 2 \mathrm{H}, \mathrm{Ar}_{3} \mathrm{H}$ ), 7.17 (dd, $J=2.0,8.7 \mathrm{~Hz}, 2 \mathrm{H}, \mathrm{Ar}_{1} \mathrm{H}$ ), 6.92 (dd, $J=$ 2.0, $8.8 \mathrm{~Hz}, 2 \mathrm{H}, \mathrm{Ar}_{3} \mathrm{H}$ ), 6.82 (dd, $J=2.0,8.7 \mathrm{~Hz}, 2 \mathrm{H}, \mathrm{Ar}_{1} \mathrm{H}$ ), 4.03 (s, 3H, OC$\underline{\mathrm{H}}_{3}$ ), 3.85 (s, $3 \mathrm{H}, \mathrm{OC}_{3}$ ), 3.80 (s, 3H, OC$\left.\underline{H}_{3}\right), 2.67$ (q, $\left.J=7.5 \mathrm{~Hz}, 2 \mathrm{H}, \mathrm{CH}_{2}\right), 1.09$ (t, $\left.J=7.5 \mathrm{~Hz}, 3 \mathrm{H}, \mathrm{C}_{3}\right) ;{ }^{1} \mathrm{H}-{ }^{15} \mathrm{~N}-\mathrm{NMR}$ $\left(\mathrm{CDCl}_{3}\right) \delta, \underline{\mathrm{N}-1}$ 201.8, $\underline{\mathrm{N}-2}$ 299.2; Elem. Anal. : C 67.96, H 5.48, N 9.14; C 68.11, H 5.53, N 9.22.

\section{3-(4-Methoxy-3-nitrophenyl)-1,5-bis-(4-methoxyphenyl)-4-propyl-1H-pyrazole (4c)}

Diketone 3c (0.2 g, 0.5mmol) was reacted with 4-methoxyphenylhydrazine hydrochloride (0.48 g, $2.5 \mathrm{mmol}$ ) for $20 \mathrm{~h}$, according to the general procedure. The resulting dark brown residue was purified by flash column chromatography (40\% EtOAc/hexanes) to provide the title product $\mathbf{4 c}$ as a pale brown solid $(0.1 \mathrm{~g}, 85 \%)$ and $0.1 \mathrm{~g}$ of the substrate 3c. M.p. 131-133 ${ }^{\circ} \mathrm{C} ;{ }^{1} \mathrm{H}-\mathrm{NMR}\left(\mathrm{CDCl}_{3}\right) \delta, 8.31(\mathrm{~d}, J=2.0$ Hz, 1H, H-2'), 8.01 (dd, $J=2.0,8.1$ Hz, 1H, H-6'), 7.68 (d, $J=8.1 \mathrm{~Hz}, 1 \mathrm{H}, \mathrm{H}-5$ '), 7.21 (d, $J=7.1 \mathrm{~Hz}$, $2 \mathrm{H}, \mathrm{Ar}_{3} \mathrm{H}$ ), $7.16\left(\mathrm{~d}, J=8.5 \mathrm{~Hz}, 2 \mathrm{H}, \mathrm{Ar}_{1} \mathrm{H}\right), 6.93$ (d, $\left.J=7.1 \mathrm{~Hz}, 2 \mathrm{H}, \mathrm{Ar}_{3} \mathrm{H}\right), 6.83(\mathrm{~d}, J=8.5 \mathrm{~Hz}, 2 \mathrm{H}$, $\mathrm{Ar}_{1} \mathrm{H}$ ), 4.04 (s, $3 \mathrm{H}, \mathrm{OC}_{3}$ ), 3.85 (s, $3 \mathrm{H}, \mathrm{OC}_{3}$ ), 3.80 (s, $3 \mathrm{H}, \mathrm{OC}_{3}$ ), 2.60 (t, $J=7.7 \mathrm{~Hz}, 2 \mathrm{H}, \underline{\mathrm{C}}_{2}$ ), $1.45\left(\mathrm{~m}, 2 \mathrm{H}, \underline{\mathrm{C}}_{2}\right), 0.84\left(\mathrm{t}, J=7.2 \mathrm{~Hz}, 3 \mathrm{H}, \mathrm{C}_{3}\right) ;{ }^{1} \mathrm{H}-{ }^{15} \mathrm{~N}-\mathrm{NMR}\left(\mathrm{CDCl}_{3}\right) \delta, \underline{\mathrm{N}-1} 202.9, \underline{\mathrm{N}-2} 300.1$; Elem. Anal.: C 68.48, H 5.75, N 8.87; C 68.61, H 5.59, N 8.75.

3-(4-Methoxy-3-nitro-phenyl)-1-(4-methoxy-phenyl)-4-methyl-5-phenyl-1H-pyrazole (7a) and 5-(4Methoxy-3-nitro-phenyl)-1-(4-methoxy-phenyl)-4-methyl-3-phenyl-1H-pyrazole (7b)

A stirred solution of compound 6 (0.33 g, $1.05 \mathrm{mmol})$ in DMF (30mL) and THF (10mL), was reacted with 4-methoxyphenylhydrazine hydrochloride (0.92 g, $5.25 \mathrm{mmol}$ ) according to the general procedure for pyrazole synthesis. Upon purification by flash chromatography (10\% EtOAc/hexanes), an inseparable mixture of the title compounds was obtained as a dark brown solid (0.16g, 69\%), while $0.15 \mathrm{~g}$ of the starting compound 6 was recovered. Semipreparative HPLC separation using $\mathrm{H}_{2} \mathrm{O} / \mathrm{CH}-$ ${ }_{3} \mathrm{CN}$ (20:80) as eluent and flow rate of $1.6 \mathrm{ml} / \mathrm{min}$, provided equal amounts of the two regioisomers (UV detector at $254 \mathrm{~nm}, \mathrm{t}_{\mathrm{R} 7 \mathrm{a}}=11.2 \mathrm{~min}, \mathrm{t}_{\mathrm{R} 7 \mathrm{~b}}=13.7 \mathrm{~min}$ ). 7a: M.p. $127-128{ }^{\circ} \mathrm{C} ;{ }^{1} \mathrm{H}-\mathrm{NMR}\left(\mathrm{CDCl}_{3}\right) \delta$, 8.42 (d, $J=2.2$ Hz, 1H, H-2'), 8.11 (dd, $J=2.2,8.7$ Hz, H-6'), 7.45 (d, $J=8.7$ Hz, 1H, H-5'), 7.28-

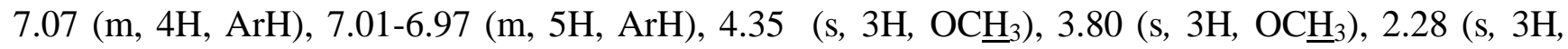
$\left.\mathrm{CH}_{3}\right) .{ }^{1} \mathrm{H}^{-15} \mathrm{~N}-\mathrm{NMR}\left(\mathrm{CDCl}_{3}\right) \delta$, N-1 203.7, $\underline{\mathrm{N}-2}$ 299.6; Elem. Anal.: C 69.39, H 5.10, N 10.11; C 69.51, H 5.02, N 10.30; 7b: M.p. $141-143^{\circ} \mathrm{C}$; ${ }^{1} \mathrm{H}-\mathrm{NMR}\left(\mathrm{CDCl}_{3}\right) \delta, 8.30$ (d, $J=2.2 \mathrm{~Hz}, 1 \mathrm{H}, \mathrm{H}-2$ '), 8.09 (dd, $J$ = 2.2, $8.7 \mathrm{~Hz}, 1 \mathrm{H}, \mathrm{H}-6$ '), 7.40 (d, $J=8.7 \mathrm{~Hz}, 1 \mathrm{H}, \mathrm{H}-5$ '), 7.37-7.14 (m, 4H, ArH), 7.08-6.89 (m, 5H,

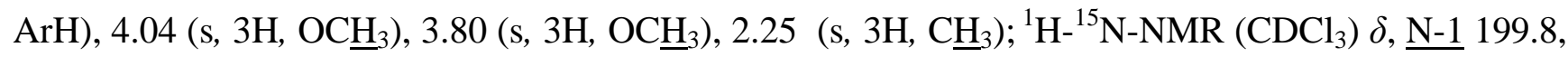
N-2 295.7; Elem. Anal.: C 69.39, H 5.10, N 10.11; C 69.67, H 4.93, N 9.94. 


\section{General Demethylation Procedure}

To a stirred solution of methyl-protected pyrazole (1 equiv) in $\mathrm{CH}_{2} \mathrm{Cl}_{2}$ at $-78{ }^{\circ} \mathrm{C}$ was added dropwise a $1 \mathrm{M} \mathrm{BBr}_{3}$ solution in $\mathrm{CH}_{2} \mathrm{Cl}_{2}$ (3-5 equiv). Upon completion of $\mathrm{BBr}_{3}$ addition, the reaction was stirred at $-78{ }^{\circ} \mathrm{C}$ for $1 \mathrm{~h}$ and then allowed to reach room temperature. Stirring was continued for additional $16 \mathrm{~h}$, then the mixture was cooled to $0{ }^{\circ} \mathrm{C}$, and carefully quenched with $\mathrm{H}_{2} \mathrm{O}(15-25 \mathrm{~mL})$ and extracted repeatedly with EtOAc. The combined organic layers were dried over $\mathrm{Na}_{2} \mathrm{SO}_{4}$ and evaporated under reduced pressure. Flash chromatographic purification using a $5 \% \mathrm{MeOH} / \mathrm{CH}_{2} \mathrm{Cl}_{2}$ mixture as the eluting solvent system provided the desired demethylation products.

\section{4-[1,5-Bis-(4-hydroxyphenyl)-4-methyl-1H-pyrazol-3-yl]-2-nitrophenol (5a)}

A stirred solution of $\mathbf{4 a}$ in $\mathrm{CH}_{2} \mathrm{Cl}_{2}$ was deprotected using $\mathrm{BBr}_{3}$ according to the general demethylation procedure. Purification by flash chromatography afforded the title compound as a tan solid (87\%). M.p. 155-156 ${ }^{\circ} \mathrm{C}$; ${ }^{1} \mathrm{H}-\mathrm{NMR}$ (acetone-D6) $\delta, 8.87$ (s, 3H, OH), 8.52 (d, $J=2.2 \mathrm{~Hz}, 1 \mathrm{H}, \mathrm{H}-$ 3’’), 8.19 (dd, $J=2.2,8.7 \mathrm{~Hz}, 1 \mathrm{H}, \mathrm{H}-5^{\prime \prime}$ ), 7.32 (d, $J=8.7 \mathrm{~Hz}, 1 \mathrm{H}, \mathrm{H}-6$ '’), 7.16 (d, $J=8.6 \mathrm{~Hz}, 2 \mathrm{H}$, $\mathrm{Ar}_{3} \mathrm{H}$ ), 7.11 (d, $\left.J=8.6 \mathrm{~Hz}, 2 \mathrm{H}, \mathrm{Ar}_{1} \mathrm{H}\right), 6.91\left(\mathrm{~d}, J=8.6 \mathrm{~Hz}, 2 \mathrm{H}, \mathrm{Ar}_{3} \mathrm{H}\right), 6.81$ (d, $J=8.6 \mathrm{~Hz}, 2 \mathrm{H}, \mathrm{Ar}_{1} \mathrm{H}$ ), 2.26 (s, 3H, $\left.\mathrm{CH}_{3}\right) . I R(\mathrm{ZnSe}) \mathrm{v}=3303 \mathrm{~cm}^{-1}$, (OH); Elem. Anal.: C 65.50, H 4.25, N 10.42; C 65.31, H 4.11, N 10.58 .

\section{4-[2,5-Bis-(4-hydroxyphenyl)-4-methyl-1H-pyrazol-3-yl]-3-nitrophenol (5d)}

A stirred solution of $\mathbf{4 d}$ in $\mathrm{CH}_{2} \mathrm{Cl}_{2}$ was deprotected using $\mathrm{BBr}_{3}$ according to the general demethylation procedure. Purification by flash chromatography afforded the title compound as a pale yellow solid (88\%). M.p. 161-162 ${ }^{\circ} \mathrm{C} ;{ }^{1} \mathrm{H}-\mathrm{NMR}$ (acetone-D6) $\delta, 8.86$ (s, 3H, OH), 8.05 (d, $J=1.9 \mathrm{~Hz}$, 1H, H-3'), 7.66 (dd, $J=1.9,8.9$ Hz, 1H, H-5'), 7.23 (d, $J=8.9 \mathrm{~Hz}, 1 \mathrm{H}, \mathrm{H}-6$ '’), 7.19 (d, $J=8.8 \mathrm{~Hz}$, $2 \mathrm{H}, \mathrm{Ar}_{3} \mathrm{H}$ ), 7.02 (d, $\left.J=8.8 \mathrm{~Hz}, 2 \mathrm{H}, \mathrm{Ar}_{1} \mathrm{H}\right), 6.95$ (d, $\left.J=8.8 \mathrm{~Hz}, 2 \mathrm{H}, \mathrm{Ar}_{3} \mathrm{H}\right), 6.83$ (d, $J=8.8 \mathrm{~Hz}, 2 \mathrm{H}$, $\left.\mathrm{Ar}_{1} \mathrm{H}\right), 2.25$ (s, 3H, $\left.\mathrm{CH}_{3}\right) . I R(\mathrm{ZnSe}) \mathrm{v}=3313 \mathrm{~cm}^{-1}(\mathrm{OH})$; Elem. Anal.: C 65.50, H 4.25, N 10.42; C 65.20, H 4.14, N 10.53.

4-[4-Ethyl-1,5-bis-(4-hydroxyphenyl)-1H-pyrazol-3-yl]-2-nitrophenol (5b)

A stirred solution of $\mathbf{4 b}$ in $\mathrm{CH}_{2} \mathrm{Cl}_{2}$ was deprotected using $\mathrm{BBr}_{3}$ according to the general demethylation procedure. Purification by flash chromatography afforded the title compound as a tan solid (74\%). ${ }^{1} \mathrm{H}-\mathrm{NMR}$ (acetone- $\mathrm{D}_{6}$ ) $\delta, 10.52$ (s, 1H, OH), 8.73 (s, 1H, OH), 8.64 (s, 1H, OH), 8.53 (d, $J=2.2 \mathrm{~Hz}, 1 \mathrm{H}, \mathrm{H}-3$ '’), 8.19 (dd, $J=2.2,8.7 \mathrm{~Hz}, 1 \mathrm{H}, \mathrm{H}-5$ '’), 7.33 (d, $J=8.7 \mathrm{~Hz}, 1 \mathrm{H}, \mathrm{H}-6$ '’), 7.16 (d, $J$ $\left.=8.6 \mathrm{~Hz}, 2 \mathrm{H}, \mathrm{Ar}_{3} \mathrm{H}\right), 7.13\left(\mathrm{~d}, J=8.6 \mathrm{~Hz}, 2 \mathrm{H}, \mathrm{Ar}_{1} \mathrm{H}\right), 6.92\left(\mathrm{~d}, J=8.6 \mathrm{~Hz}, 2 \mathrm{H}, \mathrm{Ar}_{3} \mathrm{H}\right), 6.80(\mathrm{~d}, J=8.6$ $\left.\mathrm{Hz}, 2 \mathrm{H}, \mathrm{Ar}_{1} \mathrm{H}\right), 2.69$ (q, $\left.J=7.5,12.5 \mathrm{~Hz}, 2 \mathrm{H}, \mathrm{C}_{2}\right), 1.11$ (t, $\left.J=7.5 \mathrm{~Hz}, 3 \mathrm{H}, \mathrm{C}_{3}\right) . I R(\mathrm{ZnSe}) \mathrm{v}=3283$ $\mathrm{cm}^{-1}$, (OH); Elem. Anal. : C 66.18, H 4.59, N 10.07; C 66.33, H 4.74, N 9.88. 


\section{4-[1,5-Bis-(4-hydroxyphenyl)-4-propyl-1H-pyrazol-3-yl]-2-nitrophenol (5c)}

A stirred solution of $\mathbf{4 c}$ in $\mathrm{CH}_{2} \mathrm{Cl}_{2}$ was deprotected using $\mathrm{BBr}_{3}$ according to the general demethylation procedure. Purification by flash chromatography afforded the title compound as a $\tan$ solid (60\%). ${ }^{1} \mathrm{H}-\mathrm{NMR}$ (acetone- $\mathrm{D}_{6}$ ) $\delta, 10.52$ (s, $\left.1 \mathrm{H}, \mathrm{OH}\right), 8.78$ (s, $\left.1 \mathrm{H}, \mathrm{OH}\right), 8.68$ (s, $\left.1 \mathrm{H}, \mathrm{OH}\right), 8.50$ (d, $J=2.2 \mathrm{~Hz}, 1 \mathrm{H}, \mathrm{H}-3$ '’), 8.20 (dd, $J=2.1,8.7 \mathrm{~Hz}, 1 \mathrm{H}, \mathrm{H}-5$ '’), 7.38 (d, $J=8.7 \mathrm{~Hz}, 1 \mathrm{H}, \mathrm{H}-6$ ') ), 7.16 (d, $J$ $\left.=8.3 \mathrm{~Hz}, 2 \mathrm{H}, \mathrm{Ar}_{3} \mathrm{H}\right), 7.12\left(\mathrm{~d}, J=7.7 \mathrm{~Hz}, 2 \mathrm{H}, \mathrm{Ar}_{1} \mathrm{H}\right), 6.89\left(\mathrm{~d}, J=8.3 \mathrm{~Hz}, 2 \mathrm{H}, \mathrm{Ar}_{3} \mathrm{H}\right), 6.81(\mathrm{~d}, J=7.7$ $\left.\mathrm{Hz}, 2 \mathrm{H}, \mathrm{Ar}_{1} \mathrm{H}\right), 2.65$ (t, $\left.J=7.9 \mathrm{~Hz}, 2 \mathrm{H}, \underline{\mathrm{C}}_{2}\right), 1.20\left(\mathrm{~m}, 2 \mathrm{H}, \mathrm{C}_{2}\right), 0.85$ (t, $\left.J=7.9 \mathrm{~Hz}, 2 \mathrm{H}, \mathrm{C}_{3}\right) ; I R$ $(\mathrm{ZnSe}) \mathrm{v}=3283 \mathrm{~cm}^{-1}(\mathrm{OH})$; Elem. Anal. : C 66.81, H 4.91, N 9.74; C 67.02, H 5.10, N 9.88.

\section{4-[1-(4-Hydroxyphenyl)-4-methyl-5-phenyl-1H-pyrazol-3-yl]-2-nitrophenol (8a)}

A stirred solution of $7 \mathbf{a}$ in $\mathrm{CH}_{2} \mathrm{Cl}_{2}$ was deprotected using $\mathrm{BBr}_{3}$ according to the general desmethylation procedure. Purification by flash chromatography afforded the title compound as a tan solid (70\%). ${ }^{1} \mathrm{H}-\mathrm{NMR}$ (acetone- $\mathrm{D}_{6}$ ) $\delta, 9.30$ (s, $1 \mathrm{H}, \mathrm{OH}$ ), 8.70 (s, $\left.1 \mathrm{H}, \mathrm{OH}\right), 8.40$ (d, $J=2.2 \mathrm{~Hz}, 1 \mathrm{H}, \mathrm{H}-$ 3”), 8.07 (dd, $J=2.2,8.7$ Hz, H-5”), 7.35 (d, $J=8.7$ Hz, 1H, H-6”), 7.19-7.11 (m, 5H, ArH), 7.08-6.98 (m, 4H, ArH), 4.35 (s, 3H, $\underline{\mathrm{H}}_{3} \mathrm{O}$ ), 3.80 (s, 3H, $\left.\underline{\mathrm{C}}_{3} \mathrm{O}\right), 2.28$ (s, 3H, $\left.\underline{\mathrm{H}}_{3}\right) ; I R(\mathrm{ZnSe}) \mathrm{v}=3193 \mathrm{~cm}^{-1}$ (OH); Elem. Anal.: C 68.21, H 4.42, N 10.85; C 68.44, H 4.29, N 10.71.

\section{4-[2-(4-Hydroxyphenyl)-4-methyl-5-phenyl-2H-pyrazol-3-yl]-2-nitrophenol (8b)}

A stirred solution of $\mathbf{7 b}$ in $\mathrm{CH}_{2} \mathrm{Cl}_{2}$ was deprotected using $\mathrm{BBr}_{3}$ according to the general desmethylation procedure. Purification by flash chromatography afforded the title compound as a tan solid (78\%). ${ }^{1} \mathrm{H}-\mathrm{NMR}$ (acetone- $\left.\mathrm{D}_{6}\right) \delta, 9.28(\mathrm{~s}, 1 \mathrm{H}, \mathrm{OH}), 8.69$ (s, $\left.1 \mathrm{H}, \mathrm{OH}\right), 8.25(\mathrm{~d}, J=2.2 \mathrm{~Hz}, 1 \mathrm{H}, \mathrm{H}-$ 3”), 8.02 (dd, $J=2.2,8.7$ Hz, 1H, H-5”), 7.47-7.24 (m, 4H, ArH), 7.26 (d, $J=8.7$ Hz, 1H, H-6”), 6.986.78 (m, 5H, ArH), 4.04 (s, 3H, $\mathrm{C}_{3} \mathrm{O}$ ), 3.80 (s, 3H, $\mathrm{C}_{3} \mathrm{O}$ ), 2.25 (s, 3H, $\left.\mathrm{C}_{3}\right) ; I R(\mathrm{ZnSe}) \mathrm{v}=3199$ $\mathrm{cm}^{-1}(\mathrm{OH})$; Elem. Anal.: C 68.21, H 4.42, N 10.85; C 68.39, H 4.49, N 10.71.

\section{Binding to isolated human ER $\alpha$ and $E R \beta$}

The relative binding affinity (RBA) values were assessed as previously described [22]. Briefly, the concentrations of 5a-d, 8a,b that inhibited ES2 (a fluorescein-labelled estrogen from Invitrogen) binding to isolated human ER $\alpha$ or ER $\beta$ (Invitrogen) by 50\% ( $\mathrm{IC}_{50}$ ), as assessed using a Beacon 2000 Fluorescence Polarization Reader (Invitrogen), were used to derive the RBA values of Table 1, as described in the legend to the Table.

\section{Acknowledgements}

The RBA assessment of the Estrogen Receptor by the group of Dr. Michael N. Alexis (Institute of Biological Research \& Biotechnology of the National Hellenic Research Foundation) is gratefully acknowledged. 


\section{References and Notes}

1. (a) Veeneman, G. H. Non-Steroidal Subtype Selective Estrogens. Curr. Med. Chem. 2005, 12, 1077-1136; (b) Ghosh, U.; Ganessunker, D.; Sattigeri, V. J.; Carlson, K. E.; Mortensen, D. J.; Katzenellenbogen, B. S.; Katzenellenbogen, J. A. Estrogenic diazenes: heterocyclic non-steroidal estrogens of unusual structure with selectivity for estrogen receptor subtypes. Bioorg. Med. Chem. 2003, 11, 629-657; (c) Gao, H.; Katzenellenbogen, J. A.; Garg, R.; Hansch, C. Comparative QSAR Analysis of Estrogen Receptor Ligands. Chem. Rev. 1999, 99, 723-744.

2. Compton, D. R.; Sheng, S. B.; Carlson, K. E.; Rebacz, N. A.; Lee, I. Y.; Katzenellenbogen, B. S.; Katzenellenbogen, J. A. Pyrazolo[1,5-a]pyrimidines: Estrogen Receptor Ligands Possessing Estrogen Receptor $\beta$ Antagonist Activity. J. Med. Chem. 2004, 47, 5872-5893.

3. Compton, D. R.; Carlson, K. E.; Katzenellenbogen, J. A. Pyrazolo[1,5-a]pyrimidines as estrogen receptor ligands: defining the orientation of a novel heterocyclic core. Bioorg. Med. Chem. Lett. 2004, 14, 5681-5684.

4. Magarian, R. A.; Overaacre, L. B.; Singh, S.; Meyer, K. L. Medicinal Chemistry of Nonsteroidal Antiestrogens: A Review. Curr. Med. Chem. 1994, 1, 61-104.

5. (a) Hartman, J.; Lindberg, K.; Morani, A.; Inzunza, J.; Ström, A.; Gustafsson, J.-Å. Estrogen Receptor $\beta$ Inhibits Angiogenesis and Growth of T47D Breast Cancer Xenografts. Cancer Res. 2006, 66, 11207-11213; (b) Gustafsson, J.-Å. ER beta scientific visions translate to clinical uses. Climacteric 2006, 9, 156-160.

6. Hillisch, A.; Peters, O.; Kosemund, D.; Müller, G.; Walter, A.; Schneider, B.; Reddersen, G.; Elger, W.; Fritzmeier, K.-H. Dissecting Physiological Roles of Estrogen Receptor $\alpha$ and $\beta$ with Potent Selective Ligands from Structure-Based Design. Mol. Endocrinol. 2004, 18, 1599-1609.

7. (a) Pettersson, K.; Gustafsson, J.-Å. Role of Estrogen Receptor Beta in Estrogen Action. Ann. Rev. Physiol. 2001, 63, 165-192; (b) Nilsson, S. Kuiper, G. G. J. M.. Gustafsson, J.-Å. ERß: a novel estrogen receptor offers the potential for new drug development. Trends Endocrinol. Metab. 1998, 9, 387-395; (c) Kuiper, G. G. J. M.; Enmark, E.; Pelto-Huikko, M.; Nilsson, S.; Gustafsson, J.-Å. Cloning of a novel estrogen receptor expressed in rat prostate and ovary. Proc. Natl. Acad. Sci. U.S.A 1996, 93, 5925-5930.

8. Stauffer, S. R.; Coletta, C. J.; Tedesco, R.; Nishigushi, G.; Carlson, K. E.; Sun, J.; Katzenellenbogen, B. S.; Katzenellenbogen, J. A. Pyrazole Ligands: Structure-Affinity/Activity Relationships and Estrogen Receptor- $\alpha$-Selective Agonists. J. Med. Chem. 2000, 43, 4934-4947.

9. Fink, B. E.; Mortensen, D. S.; Stauffer, S. R.; Aron, Z. D.; Katzenellenbogen, J. A. Novel structural templates for estrogen-receptor ligands and prospects for combinatorial synthesis of estrogens. Chem. Biol. 1999, 6, 205-219.

10. Stauffer, S. R.; Huang, Y. R.; Aron, Z. D.; Coletta, C. J.; Sun, J.; Katzenellenbogen, B. S.; Katzenellenbogen. J. A. Triarylpyrazoles with basic side chains development of pyrazole-based estrogen receptor antagonists. Bioorg. Med. Chem. 2001, 9, 151-161.

11. Lin, X.; Huebner, V. Non-steroidal ligands for steroid hormone receptors. Curr. Opin. Drug Disc. 2000, 3, 383-398.

12. Pezzella, A.; Manini, P.; Napolitano, A.; Crescenzi, O.; Barone, V.; d’Ischia, M. Steroids. Oxidative chemistry of 2-nitro and 4-nitroestradiol: Dichotomous behavior of radical 
intermediates and novel potential routes for oxyfunctionalization and B-ring fission of steroidal scaffolds. Steroids 2005, 70, 543-550.

13. Dubs, M.; Dieks, H.; Günther, W.; Kötteritzsch, M.; Poppitz, W.; Schönecker, B. O-Silylated steroidal cis-aminoalcohols as chiral auxiliaries: highly diastereoselective Pd-catalyzed cyclopropanation of $\alpha, \beta$-unsaturated aldimines. Tetrahedron Lett. 2002, 43, 2499-2503.

14. Davis, A. P.; Joos, J. B. Steroids as organising elements in anion receptors. Coord. Chem. Rev. 2003, 240, 143-156.

15. Mueck, A. O.; Seeger, H. Hormone Therapy after Endometrial Cancer. Horm. Res. 2004, 62, 4048.

16. Beauregard, J.-M.; Turcotte, E.; Bénard, F. Steroid receptor imaging in breast cancer. PET Clin. North Amer. 2006, 1, 51-70.

17. Brandenburger, F.; Matthes, B.; Seifert, K.; Strohriegl, P. Cholesteric polymeric networks with steroids as chiral components. Liq. Crystal. 2001, 28, 1035-1039.

18. Santaniello, E.; Ravasi, M.; Ferraboschi, P. A-Ring nitration of estrone. J. Org. Chem. 1983, 48, 739-740.

19. Toader, V.; Xu, X.; Nicolescu, A.; Yu, L.; Bolton, J.; Thatcher, G. R. Nitrosation, Nitration, and Autoxidation of the Selective Estrogen Receptor Modulator Raloxifene by Nitric Oxide, Peroxynitrite, and Reactive Nitrogen/Oxygen Species. Chem. Res. Toxicol. 2003, 16, 1264-1276.

20. Palomino, E.; Heeg, M. J.; Pilat, M. J.; Hafner, M.; Polin, L.; Brooks, S. C. Crystal structure, receptor binding, and gene regulation of 2- and 4-nitroestradiols. Steroids 1996, 61, 670-676.

21. Alberola, A.; Andres, C.; Gonzalez Ortega, A.; Pedrosa, R. Regiospecific synthesis of 1arylpyrazoles. J. Heterocycl. Chem. 1984, 21, 1575-1576.

22. Fokialakis, N.; Lambrinidis, G.; Mitsiou, D. J.; Aligiannis, N.; Mitakou, S.; Skaltsounis, A. L.; Pratsinis, H.; Mikros, E.; Alexis, M. N. A New Class of Phytoestrogens: Evaluation of the Estrogenic Activity of Deoxybenzoins. Chem. Biol. 2004, 11, 397-406.

Sample Availability: Available from the authors.

(C) 2007 by MDPI (http://www.mdpi.org). Reproduction is permitted for noncommercial purposes. 